\title{
A High-speed Adaptive PMD Compensation Scheme Based on DSP Using DPSO Algorithm
}

\author{
Jinnan Zhang, Xueguang Yuan, YangAn Zhang, Minglun Zhang ,Xiaoguang Zhang \\ Key Laboratory of Information Photonics and Optical Communications, Ministry of Education, \\ Beijing University of Posts and Telecommunications, Beijing 100876, China \\ Phone: +(86)01062284004, Fax: +(86)01062284004-82, Email:jinnanjinnan@gmail.com
}

\begin{abstract}
This paper describes implementation of a DSP for adaptive PMD compensation in $80 \mathrm{Gbits} / \mathrm{s}$ DQPSK optical fiber communication systems. Using dithering particle swarm optimization (DPSO) algorithm, the searching process and tracking process could be completed in $10 \mathrm{~ms}$ and $1 \mathrm{~ms}$ respectively.

\section{Introduction}

Polarization mode dispersion (PMD) causes time and channel dependent pulse distortions in high-speed optical communication systems, potentially limiting their transmission capacity [1,2]. The adaptive PMD compensation has been a hot topic in recent years. The techniques of monitoring PMD in fiber links and control algorithm are most important factors for a PMD adaptive compensation system. In this paper, we report an experiment of adaptive PMD compensation in 80Gbits/s DQPSK system, in which a three-stage adaptive PMD compensator was used. DPSO algorithm was introduce as control algorithm for the first time,which showed powerful ability of fast searching global optimum without sinking in local sub-optima. We completed 1order PMD and 2-order PMD compensation.
\end{abstract}

\section{Experiment Setup}

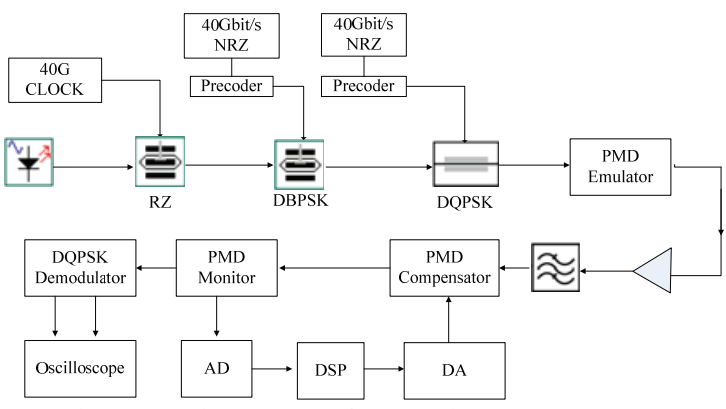

Fig.1 Experiment setup for adaptive three-stage PMD Compensation

The experiment setup is shown in Fig.1. 80Ghit/s $2^{23}-1$ of DQPSK formats are produced at the wavelength $1551 \mathrm{~nm}$. The PMD emulator consists of two-section units of two manually adjusted polarization controllers (PC1 and $\mathrm{PC} 2$ ) and two polarization maintaining fibers pieces (PMF1 and PMF2) of 60ps and 20 ps DGD. An optical band-pass filter with $3 \mathrm{~dB}$ bandwidth of $0.8 \mathrm{~nm}$ is used to suppress ASE noise form EDFA. For three-stage PMD compensator, each stage has an electrically controlled polarization controller and a delay line. For the electrically polarization controllers, each of them has four fiber-squeezer cells to be adjusted with $0-10 \mathrm{~V}$ voltage, out of which only three cells are used for PC3 and PC4, one cell for PC5 in the experiment. Therefore there are seven voltages to he controlled, which is 7 degrees of freedom (DOF) problem. The delay lines PMF3, and PMF4 are with DGDs of 20ps and 40ps respectively. As the PMD monitor, an in-line polarimeter is used to measure instantaneously the Stokes parameters S0, S1, S2, S3 and DOP in fiber link. The logic control unit adjusts the voltages of seven cells of PC3, PC4, and PC5 to searching the optimum of the whole fiber link by using DPSO algorithm according to the DOP feedback signals, as shown in Fig.2. The effectiveness of PMD compensation is observed by eye diagram on the oscilloscope. In our scheme, AD7933 was choosen to implement four simultaneous-sampling from an in-line polarimeter in the PMD monitoring unit. And DA chip TLC7225 output three voltage signals to adjust three degrees of freedom for each PC. we use the TMS320VC33 DSP as signal processor.

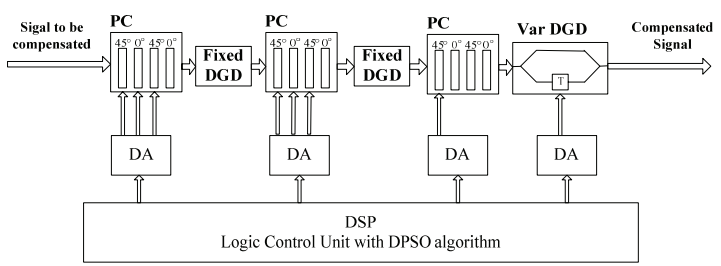

Fig.2. The configurations of three-stage compensators.

\section{DPSO Algorithm}

In the logic control unit we adopt dithering particle swarm optimization algorithm (DPSO) as the control algorithm. The particle swarm optimization (PSO) algorithm is a new community intelligence optimization method firstly proposed by Kennedy and Eberhart in 1995 [3]. To be exactly similar with other global optimization algorithm, the PSO algorithm tends to suffer from premature convergence.

In order to overcome the problem of premature convergence, many improvements have been suggested $[4,5]$. DPSO method proposed in this paper is also one of them. The main principle of this method is adding a new dithering method to PSO to improve the performance of it. Before particles update their position, they dither around their previous best position and attain the position of a better function value. Then we update the particles' positions using PSO algorithm, and compare the fitness of the new present best positions with the fitness of the better position got by dithering and choose a best position as the present global best 
position. We can find the function optimal solution and best position by utilizing the iteration operation of the principle. The flow chart of DPSO method is showed by Fig.3.

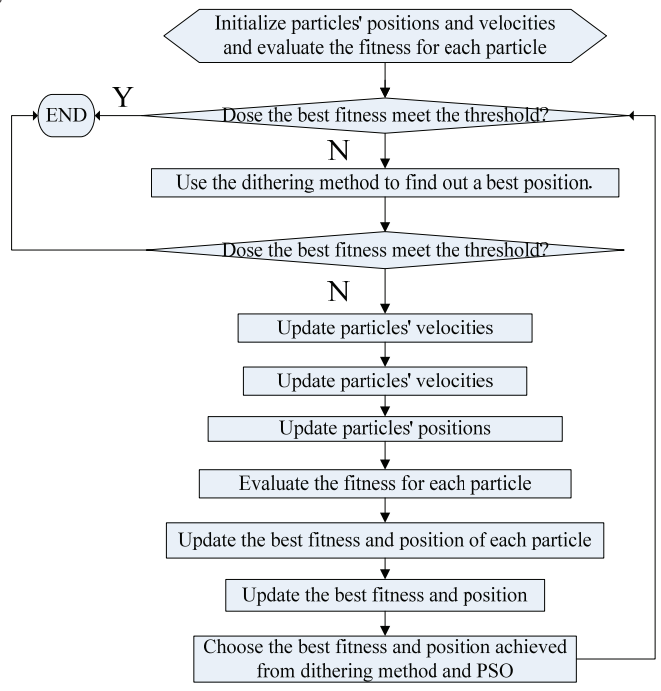

Fig.3 The flow chart of DPSO method

\section{Experiment Results}

We got a data table achieved from the adaptive PMD compensation experiment depicting the relationship between DOP and variable voltages of the polarization controller. Then, we tested DPSO and PSO algorithm using this data table by simulation. We regarded DOP as the fitness, and variable voltages as arguments. Our aim was to find the best DOP in the data table. We conducted 50 times of the simulation, and the max iteration for each time was 50. Fig.4 shows the relationship between the best DOP we find and the iteration.

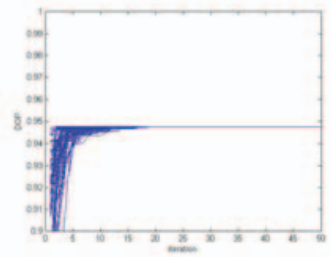

(a)

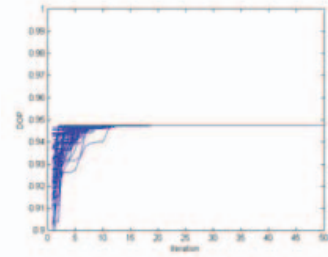

(b)
Fig.4 The best DOP vs. iteration (a) PSO; (b) DPSO

The simulation shows both two methods have found approximately the same DOP and position. Also, from Fig 4 we can get some conclusions: particles from both of the two methods have not been locked into the local sub-optima; both of the two methods get the precise results in 50 times.

Then we set a threshold and conduct the program for 50 times. For each time, when the threshold is met, the search will be ended and the final iteration number will be recorded. Fig 5. shows the relationship between the number of the corresponding iteration records and the iteration. From Fig.5 we can get the mean convergence iteration number. The mean convergence iteration number of PSO algorithm is 3.64 , and that of
DPSO method is 2.66. So the speed of DPSO method is higher than that of PSO algorithm.

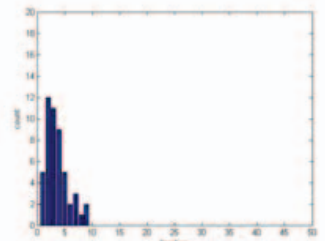

(a)

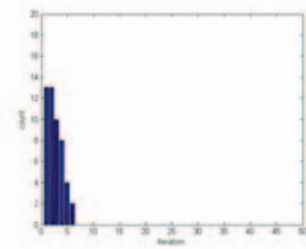

(b)
Fig.5 The number of the corresponding iteration records vs. Iteration (a) PSO; (b) DPSO

We also compared two algorithms through experiment. Defining the time slot from the previous $\mathrm{D} / \mathrm{A}$ to the next D/A as one time unit, we can use this time unit to measure the response time of the compensator. The compensation performance of the PMD adaptive compensator using both algorithm was tested. One time unit in our experiment is equivalent to about 40us. The searching process and the tracking process for automatic PMD compensation using DPSO algorithm could be completed in less than $10 \mathrm{~ms}$ and $1 \mathrm{~ms}$ respectively while the searching process and the tracking process using PSO algorithm need about $14 \mathrm{~ms}$ and 1.2ms. Experiment results is shown in Fig.6.

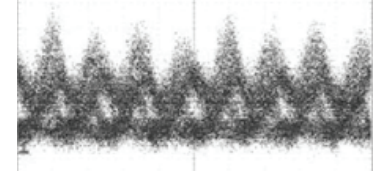

(a) Without compensation

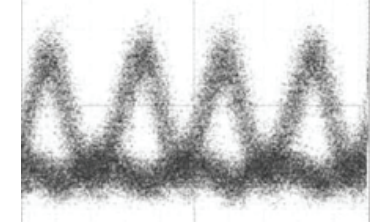

(b) With compenstion

\section{CONCLUSION}

We demonstrated a successful experiment of highspeed adaptive PMD compensation based on DSP with three-stage compensator in 80Gbit/s DQPSK system. The PMD compensation captivity is $90 \mathrm{ps}$ at the OSNR of $2 \mathrm{~dB}$. A new PSO algorithm, the DPSO method, combined with a new dithering method algorithm, was introduced as control algorithm, which was very suitable for multi-dimensional problem in PMD compensation. The result shows DPSO method is better than PSO algorithm in the convergent speed.

\section{REFERENCES}

[1] H. Kogelnik, R. M. Jopson, and L. Nelson, "Polarization-mode dispersion in Optical Fiber Telecommunications IV B", I. Kaminow and T. Li, Eds. New York: Academic, 2002.

[2] M. Shtaif and M. Boroditsky, "The effect of the frequency dependence of PMD on the performance of optical communications systems" IEEE Photon. Technol. Lett., vol. 15, no. 10, pp. 1369-1371, Oct. 2003.

[3] J.Kennedy, R. Eberhart, "Particle Swarm Optimization", Proc. on Feedback Mechanism, IEEE Int'l. Conf. on Neural Networks, vol.VI, IEEE Service Center, 1995, pp. 1942 - 1948.

[4] Thiemo Krink, Jakob S VesterstrOm, and Jacques Riget, "Particle Swarm Optimization with Spatial Particle Extension", Proceedings of the 2002 Congress on Evolutionary Computation, vol.2, 2002, pp. $1474-1479$.

[5] Xiaofeng Xie, Wenjun Zhang, "Dissipative Particle Swarm Optimization", Proceedings of the 2002 Congress on Evolutionary Computation, CEC'02, vol.2, 2002, pp. 1456 - 1461. 